\title{
Axillary lymph node surgical treatment
}

\author{
Daniela Andreina Terribile ${ }^{1}$, Cristina Accetta ${ }^{1}$, Sabatino D'Archi ${ }^{1}$, Ida Paris ${ }^{2}$, Danilo Di Giorgio ${ }^{1}$, Giorgia \\ Garganese $^{1}$, Simona Maria Fragomeni ${ }^{1}$, Riccardo Masetti ${ }^{1}$
}

${ }^{1}$ Multidisciplinary Breast Center, ${ }^{2}$ Division of Gynecologic Oncology, Fondazione Policlinico Universitario Agostino Gemelli, Università Cattolica del Sacro Cuore, Rome 00136, Italy

Contributions: (I) Conception and design: DA Terribile, C Accetta, S D'Archi; (II) Administrative support: None; (III) Provision of study materials or patients: All authors; (IV) Collection and assembly of data: All authors; (V) Data analysis and interpretation: DA Terribile, C Accetta, S D'Archi; (VI) Manuscript writing: All authors; (VII) Final approval of manuscript: All authors.

Correspondence to: Daniela Andreina Terribile, MD. Multidisciplinary Breast Center, Fondazione Policlinico Universitario Agostino Gemelli, Università Cattolica del Sacro Cuore, Rome 00136, Italy. Email: daniterribile@gmail.com.

\begin{abstract}
Nowadays, the overall attention is focused on de-escalating treatments for breast cancer (BC) including surgery, radiotherapy and chemotherapy. The introduction of sentinel lymph node biopsy (SLNB) has led to less invasive surgical approaches for accurately staging the axilla, with axillary lymph node dissection (ALND) progressively confined to a limited group of patients. One of the goal of surgery in de-escalating approaches is to reduce surgical morbidity by restricting or avoiding axillary surgery with no effect on survival. In this context the importance of imaging study for preoperative identification of axillary metastasis, in order to reduce axillary surgery, is gradually improving while the role of intraoperative assessment of sentinel nodes is progressively becoming limited to restricted groups of patients. According to the results of the ACOSOG Z0011 and following the most important guidelines, ALND can be safely omitted in selected patients treated with breast conserving surgery (BCS) with one or two positive SLNB while the adoption of SLNB positive alone in patients undergoing mastectomy is not yet defined. The increased employment of neoadjuvant chemotherapy (NAC) and the use of SLNB in patients after NAC plays an important role in de-escalation of axillary surgery in this group of patients. However current studies on this topic are still controversial, mainly about clinically positive lymph nodes $(\mathrm{cN}+)$ pre NAC patients or how to manage positive SLNB in post NAC patients. Some authors have collected predictive factors of positive non sentinel lymph nodes (NSLNs) in nomograms, considered an useful tool to avoid unnecessary further surgery. Elderly women represent specific group of patients where the axillary approach needs to be properly resized. The management of axilla in BC is in continuous evolution and ongoing studies could make even SLNB useless in the next future.
\end{abstract}

Keywords: Breast cancer (BC); axillary management; sentinel node biopsy; axillary lymph node dissection (ALND); neoadjuvant therapy

Submitted Jul 21, 2017. Accepted for publication Sep 05, 2017.

doi: $10.21037 /$ tcr.2017.09.19

View this article at: http://dx.doi.org/10.21037/tcr.2017.09.19

\section{Introduction}

In the last years the role of surgery in breast cancer (BC) treatment and axillary staging is gradually changing. The surgical management of $\mathrm{BC}$ is continuously evolving towards less extensive surgery following a new concept of "de-escalating surgery". The axillary lymph node dissection
(ALND), considered for more than a century as a milestone of treatment for all BC patients, was upset-down in the last 15 years. With the introduction and worldwide spread of sentinel lymph node biopsy (SLNB) a new era began.

The use of SLNB replaced the ALND in patients with a clinically node negative status, accurately staging the axilla 
without compromising regional recurrence rates and overall survival and markedly reducing the morbidity of ALND.

Actually, several studies analyzed the possibility of omitting ALND in certain patient groups with SLNB metastases with a subsequent decline in the indication for completion ALND, considering also the efficacy of adjuvant therapies to reach an acceptable local control of the disease.

In this article we review guidelines and literature regarding the current axillary management. We focused our research on main different subset as: the role of preoperative axillary evaluation; changes in intraoperative assessment of sentinel nodes; how to approach the axilla in case of positive SLNB during breast conservative surgery (BCS) or mastectomy; utilization of SLNB in neoadjuvant chemotherapy (NAC) setting; nomograms and predictive factors of additive positive non sentinel lymph nodes (NSLNs); management of the axilla in elderly women and future ongoing studies.

\section{Preoperative axillary imaging}

With the common adoption of mini-invasive axillary staging, efforts are now focusing on preoperative identification of axillary metastasis given that they may have a direct impact on surgical and medical approaches, from NAC to breast reconstructive options (1). Currently both physical evaluation and breast-directed imaging modalities are being used to test the presence and evaluate the extent of the disease. The imaging modalities run from ultrasound (US) and US guided fine needle aspiration cytology (US-FNA) to magnetic resonance imaging (MRI) (2) and 18F-Fluorodeoxyglucose-positron emission tomography/computed tomography $\left({ }^{18} \mathrm{~F}-\mathrm{FDG}-\mathrm{PET} / \mathrm{CT}\right)$. The ACOSOG Z0011 trial identified clinically nodenegative patients by physical exams alone (3). However, given that physical examination has poor sensitivity (35\% to $41 \%$ ) and high false-positive rates (53\%) with regards to the axilla, attention has recently shifted towards axillary imaging (4). As a result, the use of preoperative axillary US integrated with fine needle aspiration cytology (FNA) has become much more frequent in $\mathrm{BC}$ patients (5) as a fast, non-surgical, staging procedure associated with a low rate of complications (6-8). Several studies report of a sensitivity of US-FNA in the preoperative staging of BC of up to $80 \%$ with $100 \%$ specificity (9-11). Boland et al. (12) showed that the use of preoperative axillary US and USFNA is successful in identifying a cohort of patients with a higher burden of axillary nodal disease to address directly to ALND or NAC. Despite other researches have suggested that axillary imaging with or without USFNA may be beneficial to allocate high-risk node-positive patients directly to ALND (10,13-16), Pilewskie et al. (17) showed that in a population of patients meeting ACOSOG Z0011 criteria, axillary imaging (MRI, mammogram and US) did not reliably identify patients with $>2$ positive sentinel lymph nodes, who should undergo ALND without determination of the number of involved nodes. According to the mentioned study, if all patients with abnormal axillary imaging were triaged to ALND, the $68-73 \%$ would have been over-treated and subjected to unnecessary procedures. Y L et al. (18) evaluated a group of patients not fulfilling ACOSOG Z0011 inclusion criteria, demonstrating that positive US-FNA is very accurate in predicting macroscopic impacts of axillary nodes. Patients that did not fall within the ACOSOG Z0011 parameters can move on to axillary clearance without SLNB nor risk of over-treatment. Promoting the above evidence, the data of van Wely et al. (16) reported that patients with axillary metastases diagnosed by US-FNA have significantly more affected nodes than SLNB-positive patients and are most likely to benefit the most from further axillary treatment.

Following the possible results of SOUND trial (19) and BOOG 2013-08 trial (20), an accurate pre-operatory axillary evaluation with US or US-FNA may also lead to avoid SLNB performance in selected groups of patients with early BC.

While US is actually the standard preoperative imaging procedure, several studies are evaluating the role of MRI in axillary staging. Assing et al. (21) in their study added MRI to US in preoperative staging concluding that MRI could be useful in identification of those lymph nodes not identified on US; Arslan et al. (22) in a recent study showed that MRI has a sensitivity of $84.7 \%$ for detecting axillary lymph node metastases. Nevertheless, there is actually no evidence of the possibility of avoiding SLNB using MRI.

${ }^{18} \mathrm{~F}$-FDG-PET/CT is commonly applied in breast oncology imaging. However, the role of PET/CT in the regional staging of $\mathrm{BC}$ remains unclear, despite it being more accurate than conventional imaging modalities (23). Latest reviews reported a sensitivity and specificity that ranged from $57 \%$ to $100 \%$ and $66 \%$ to $100 \%$, respectively (24). Several authors $(23,24)$ showed that ${ }^{18} \mathrm{~F}$-FDG-PET/CT is not likely to replace SLNB for axillary assessment. Positive FDG uptake in the axilla does not always indicate axillary metastasis (15\% false-positive). Therefore, US-FNA is useful to avoid unnecessary ALND in patients with positive 
FDG uptake. On the other hand, SLNB is needed in patients with negative FDG axilla uptake and in those with negative fine-needle aspiration cytology (FNAC) of the axillary lymph nodes.

The role of the preoperative axillary imaging is in continuous evolution to help to better define the axillary management.

\section{Axillary evaluation following NAC}

Fifty-five percent of node-positive $\mathrm{cN}+\mathrm{BC}$ are downstaged by NAC (25). In order to facilitate the identification of the sentinel nodes and reduce the false negative rate (FNR), recent studies are focusing on the importance of identification of positive sentinel nodes after NAC (25-28). It is done by placing a clip in the positive node at initial diagnosis with confirmation of clipped node resection at surgery (25) or using iodine marked seed (26). The ACOSOG Z1071 trial (27) was designed to determine if SLNB was accurate in staging the axilla after chemotherapy in patients presenting with node-positive disease. The trial reported a FNR of $12.6 \%$. In this trial, where the estimated group of patient had a clip placed in nodes after needle biopsy and who had documented removal of the clipcontaining SLN, FNR was $<7 \%$. According to this data Caudle et al. (28) found that performing targeted axillary dissection, which involves removing SLNB with clipped node identified pre-therapy as containing metastatic disease, FNR was $2 \%$ versus $10.1 \%$ for SLNB alone. Koolen et al. (26) associated PET/CT before NAC with axillary lymph node marking with radioactive iodine seeds (MARI procedure) after NAC avoiding $74 \%$ of ALND in a cohort of axillary node-positive patients after NAC. The NCCN guidelines (29) have incorporated a comment that marking lymph nodes to document their removal is one method to decrease the FNR of SLNB post NAC.

\section{Intraoperative assessment of sentinel nodes}

Traditionally, intraoperative diagnosis of SLN can prevent unnecessary ALND when negative, and can allow ALND in the same surgical procedure when positive, thus avoiding a second surgery and decreasing the patient's associated discomfort (30,31). Nevertheless, according to the evolution of surgical approach in case of positive SLNB, intraoperative assessment of lymph nodes could confuse surgeons to define the better treatment planning. Moreover, there is increasing evidence that omitting ALND with or without radiotherapy in selected patients groups results in excellent and similar regional control compared to ALND. Therefore there are several reasons why the use of intraoperative assessment should be questioned. Following the results of the ACOSOG Z011 (3) and according to the American Society of Clinical Oncology (ASCO) recommendations (32), BC patients with clinically nodenegative disease and one or two positive SLNs can be safely treated with BCS and radiotherapy omitting ALND. In this patient, intraoperative assessment of SLNs could not be useful nor performed.

More controversies exist for $\mathrm{cN} 0$ patients treated with mastectomy, ASCO recommend that patients with a SLN+ tumor who are treated with mastectomy should still be offered ALND, as radiotherapy is not routinely administered in these patients. In this group of patients the intraoperative assessment of SLNs could be performed. Several studies suggested ALND may be safely omitted in patients who are treated with mastectomy with al low tumor burden in SLNs $(33,34)$. About patients undergoing NAC, according to NCCN guidelines (29) intraoperative assessment of axillary lymph node should be performed, since these patients are supposed to be treated with ALND in case of positive SLN. Van der Noordaa et al. (35) found that omitting axillary lymph node intraoperative assessment might be a reasonable option in patients presenting with limited (cN1) axillary disease and a tumor-positive lymph node while in patients with extensive nodal disease intraoperative assessment of axillary lymph nodes should be performed, since these patients should be treated with ALND in case of residual disease after NAC.

In conclusion, the use of intraoperative assessment should be limited for patients who still have an indication for ALND in case of a tumor-positive axillary lymph node. This group is made up of patients with extensive axillary disease who undergo NAC and remain node-positive after NAC and of clinically N0 patients, not treated with NAC, presenting with intraoperative unexpected extensive axillary disease.

In terms of SLN intraoperative analysis, common techniques that are used to identify tumor deposits, including touch imprint, crash, and cytological smear preparations, as well as frozen sections, all lack a sufficient degree of sensitivity (36). In this context, advances in diagnostic technology based on molecular methods for the analysis of SLN might be essential to improve therapeutic 
management.

One Step Nucleic Acid Amplification (OSNA) is a highly sensitive, automated and rapid assay that analyses lymph nodes for identifying metastases by detection and amplification of the cytokeratin 19 (CK19) mRNA, an epithelial marker in BC cells (37). Several studies demonstrated that the NSLN macro-metastatic rate increased in proportion to CK 19 mRNA copy numbers (38). The findings of Banerjee et al. (31), PiñeroMadrona et al. (39), Peg et al. (40) were consistent with Nabais et al. (38), who observed that using OSNA technique to evaluate SLN, NSLN metastases can be predicted intraoperatively, supporting in decision for ALND.

Nevertheless, in current standard clinical practice, where the use of intraoperative assessment of axillary lymph nodes is decreasing, all intraoperative techniques, including OSNA, are less employed than in the past.

\section{Breast conservative surgery and positive SLNB}

As recommended by ASCO guidelines (32) about SLNB in patients with early breast cancer (EBC), a negative SLNB has not to be followed by ALND, independently from the performed surgery type. The need for ALND after positive SLNB in BCS (breast conservative surgery) was questioned. The American College of Surgeons Oncology Group Z0011 (3) trial has been a milestone in axillary treatment in EBC with clinically negative lymph nodes but positive SLNB, in patients treated with BCS, followed by whole breast irradiation (WBI) and systemic therapies. Giuliano et al. showed that loco-regional recurrence rates was not improved by ALND, in patients with axillary tumor burden from low to moderate, compared to SLNB alone. Several criticisms have been raised up to this trial (41), as early enrollment closure with low statistical power, no respect of inclusion criteria, lost data at follow up. Although with these limits, lots of studies have been developed reporting good results in the applicability of ACOSOG Z0011 criteria in clinical practice. It led to the inclusion of those criteria in NCCN international guidelines (29). Verheuvel et al. (42) have reported their experience, with an applicability in more than $51 \%$ patients. Voutsadakin and Spadafora (43) concluded to consider omitting ALND only in patients meeting ACOSOG Z0011 criteria, that means "only in post-menopausal patients, ductal invasive carcinomas, clinically negative axilla, no extranodal extension, and estrogen receptor/progesterone receptor positive disease".

Questions have been raised about the number of sentinel lymph nodes to remove to gain a predictive value on disease free survival and to guide to perform an ALND. According to retrospective and prospective studies applying ACOSOG Z0011 criteria (44-46), the ideal number of sentinel lymph nodes to be harvested is three, since the involvement of more than three nodes requires an ALND for a high risk of residual axillary disease.

Subhedar et al. (47) proved there was no relation between the increase of number of sentinel lymph nodes removed after ACOSOG Z0011 trial and a significant decrease of ALND. They reported a higher number of lymph node removed only in younger patients and/or with larger tumors.

Yao et al. (48) underlined in their retrospective analysis the spread of approach of SLNB without ALND (National Cancer Data Base, 1998-2011), but they also reported ALND as preferred treatment in clinical practice for high risk patients (i.e., HER2-positive disease, triple negative BC and/or age $<50$ years at diagnosis). In contrast with this preference, Chung et al. (49) focused on applicability of ACOSOG Z0011 criteria in high risk patients, showing ALND can be avoided in $84 \%$ of cases with clinically negative nodes. Wang et al. (50) confirmed oncological safety of SLNB only in early lobular invasive cancer (about $7 \%$ of ACOSOG Z0011 population).

In September 2016, Giuliano et al. (51) published an update of ACOSOG Z0011 trial after a follow up of 9.25 years. In this article, they confirmed previous results about locoregional disease control, answering critical issues and pointing out about some limits of their study.

As reported by Tsao et al. (52) in their comparison of spread of SLNB according to ACOSOG Z0011 trial and after diffusion of regional guideline for ALND, in the clinical practice the trend of decrease of completion axillary clearance depends on a multifactorial and individualized decision making process. Particularly, criteria not included in Z0011 trial were patient's age, presence of extracapsular invasion (ECI), lymph node ratio (defined as the total number of positive nodes/total number of nodes dissected during SLNB), size of SLNB metastasis, ER status, type of surgery.

Jagsi et al. (53) answered to criticism about radiotherapy (RT) lacking planning data in ACOSOG Z0011, underlining the RT contribution in control of local recurrence in positive SLNB not followed by ALND.

The AMAROS trial (54) confirmed the efficacy of axillary irradiation compared to ALND after positive SLNB, having an equivalent 5 -years axillary recurrence free 
survival for T1-2 primary cancer, with a significantly less morbidity in the arm treated with RT.

Some limits were ascribed to AMAROS trial, such as small recruitment, choice of tumors with low risk of recurrence, a high number of micro-metastasis and isolated tumors cells. The Hungarian OTOASOR trial (55) have proved AMAROS effectiveness applying criteria on a larger population with eight years follow up, reporting similar axillary recurrence rate, overall survival and disease free survival in patients treated with ALND or RT after positive SLNB.

ALND or RT after positive SLNB should be considered also if sentinel lymph node is affected by an ECI $>2 \mathrm{~mm}$.

As remembered by Gooch et al. (56), ECI could be present in about $19-26 \%$ of SLNB and in about $30 \%$ of cT1-2, cN0 BC. Gooch conducted analysis on a prospective database, considering ACOSOG Z0011 criteria. They found out that ECI $>2 \mathrm{~mm}$ was correlated with a greater axillary burden, an older age, larger, multifocal and HR positive tumors, and presence of lymphovascular invasion (LVI). These evidences suggest that ECI $>2 \mathrm{~mm}$ in SLNB should guide to perform an ALND or RT also in patients with less than three positive lymph nodes. Also for Choi et al. (57), an additional RT should be considered in ECI >2 $\mathrm{mm}$, since it's related with $\mathrm{N} 2$ disease.

The first results from the German-Austrian IntergroupSentinel-Mamma (INSEMA) (58) trial have recently been published. This study is based on a double randomization in BCS for EBC: the first one to perform or not SLNB, the second one in patients with one or two positive SLNB to perform or not an ALND. A preoperative US evaluation is always performed as in the SOUND trial, with which primary and secondary endpoints are shared. With a good detection rate of the sentinel lymph node (99.5\%), at June, 2016 the first randomization has reported only $1.3 \%$ of patients with more than three involved nodes and $12.9 \%$ of patients with one or two macro-metastasis. According to the outcome, a limited recruitment was registered till now to a second randomization, also due to patients' refusal (20\% of population) and delayed recruitment by Austrian centers. A follow-on INSEMA project will be led minimizing breast irradiation in low risk patients without SLNB, while raising question is about avoiding SLNB also in patients treated to mastectomy.

As evolution of an extremely less aggressive axillary approach, great expectation are put over the ongoing prospective Italian SOUND trial (19). Authors question about role of SLNB today, since the setting of adjuvant treatment are tailored to the biological tumor characteristics, that are related to the disease prognosis more than the knowledge of axillary involvement.

All patients treated with BCS + WBI have an US axillary evaluation and a core biopsy or fine needle aspiration of suspicious lymph nodes. Population is randomized in two arms, SLNB or axillary observation. First results (59) about quality of life are about a significant less rate of disability in the observation group, more evident one week after operation (24\% in SLNB arm, 10.6\% in observation one).

\section{Mastectomy and positive SLNB}

ACOSOG Z011 trial has considered EBC that underwent only to conservative treatment. Nevertheless mastectomy is also another surgical option used in EBC with an unfavorable ratio breast size/tumor size or in multifocal tumors.

Current guidelines indicate ALND as standard of care after positive SLNB in mastectomy, but it starts to be a controversial issue.

Fu et al. (60) and Kenny et al. (61) retrospectively investigated about the applicability of ACOSOG Z0011 in patients with positive SLNB and mastectomy. Kenny et al. showed the increasingly trends to avoid ALND also in mastectomy in the post ACOSOG Z0011 era in EBC with low burden axillary disease.

$\mathrm{Fu}$ et al. concluded overall survival and systemic relapse free survival rate were the same between groups treated with ALND or RT after SLNB, and reported fewer side effects with RT than with ALND.

Miller et al. (62) reported the potential benefit in terms of sequelae of applying ACOSOG Z0011 criteria to mastectomy patients. The two years lymphedema incidence was of $2.19 \%$ after SLNB, $10.0 \%$ after SLNB + RT, 19.3\% after ALND, 30.1\% after ALND + RT.

To avoid complete axillary clearance and considering ALND in mastectomy as standard of care after positive SLNB, Cowher et al. (63) elaborated a mixed technique, called Conservative Axillary Regional Excision (CARE). With a median of 8 nodes removed and a $0.5 \%$ of local recurrence at 5 years follow up, they considered their CARE a safety conservative treatment of axilla.

More recently, FitzSullivan et al. (64) verified retrospectively outcomes of positive SLNB in mastectomy underwent to ALND or axillary RT or simple observation. At a follow up of 10 years, the higher axillary recurrence was registered in patients without treatment $(3.8 \%)$, the less 
one in the ALND group (1.6\%), the lower in patients had made axillary RT after SLNB (1.8\%). These differences were not statistically significant. The authors suggested to consider SLNB not followed by ALND only in low risk groups, such as elderly patients, with small tumor size and/ or small metastasis node size, with fewer positive lymph nodes and absence of LVI or ECI.

\section{Future knowledges from trial ongoing}

Further knowledges on axillary management could be available in future from results deriving from randomized controlled trials that are now ongoing:

(I) The Italian SINODAR ONE (65), and the English POSNOC (66) trials were both design to understand if ALND could be avoided in patients with onetwo positive SLNB, including mastectomy; a preoperative US evaluation of axillary is mandatory in both studies;

(II) The Dutch BOOG 2013-07 trial (67) is a randomized multicentric study that analyzes cT1-2 cN0 BC, with maximum three positive sentinel lymph nodes; the aim of this study is to confirm the safety due to avoiding axillary treatment (i.e., ALND or axillary irradiation) in mastectomy after positive SLNB, comparing one group of patients treated with only SLNB with the other group undergone to axillary treatment;

(III) The Swedish SENOMAC (68) trial includes in the randomization to ALND or no further surgery after positive SLNB also T3 tumors, patients undergone to neoadjuvant systemic treatment and male patients.

\section{Role of sentinel node biopsy in neoadjuvant setting}

Usually, NAC is provided with the aim of down-staging. The main objective is to reduce the tumor volume both in breast and axilla making breast and axillary conserving surgery feasible and safe (69).

Mamounas and colleagues in 2016 described the axillary pathological complete response (pCR) in triple negative (TN) and Her2 positive BCs. They showed a pCR rate of $30 \%$ with anthracycline and $40 \%$ with taxane. In case of Her2 positive BCs, the addition of Trastuzumab is related to axillary pCR rates up to $70 \%$ (70). However, performing ALND when a pCR is documented, so without active axillary disease, should be considered as a failure of treatment planning and a kind of overtreatment (71). In addition to the debate on feasibility and safety of SLNB in neo-adjuvant setting, the timing is still a topic of discussion with pros and cons (72).

Ozmen et al. (73) suggest that staging the axilla before NAC let surgeons overcome the effects of systemic therapy, reducing the number of patients referred to ALND after treatment. Furthermore, many authors $(27,70,72,74)$ described how the accuracy of SLNB may be potentially influenced by chemotherapy.

Pilewskie and Morrow (74) indicated how in several studies the most frequent concerns were related to fibrosis of lymphatic system and to the risk that a variability in axillary response may determine a FNR higher than that usually accepted. They showed the accuracy of this procedure was mostly reduced in case of bulky nodes or poor axillary response. Clearly came out how a better patients' selection is associated with the feasibility of the technique after NAC.

In fact, Mamounas and colleagues (75) examined the role of NAC analyzing the most relevant trials. The NSABP-B27, comparing NAC to adjuvant treatment and GANEA study which evaluated the role of SLNB followed by ALND in neoadjuvant setting showed encouraging data as a global identification rate of $86.5 \%$ and FNR of $10.9 \%$. These results are substantially comparable with SLNB up front.

Furthermore, two meta-analyses confirmed the accuracy of SLNB post NAC with identification rates of $90 \%$ and $89.6 \%$ or FNR of $12 \%$ and $8.4 \%$ respectively (73).

Two more meta-analysis, cited by Rubio (72) (conducted by van Deurzen and Tan) confirmed FNR comparable to SLNB performed without NAC (10.5\% and $7.4 \%)$. Regarding the identification rate, in clinically node negative patients who underwent NAC values are similar to SLNB up front (92.7\% from 5 studies, 266 patients and 94.3\%) (72).

Given the several data available in literature, in the UK guidelines the surgeons are allowed to perform a post NAC SLNB if axillary lymph nodes were considered negative by US and/or FNA before starting treatment.

The decision on the opportunity to perform the SLNB should be taken in a multidisciplinary meeting at the time of the treatment planning (71). What remains unclear is the appropriate behavior in case of pCR to treatment. Therefore, SLNB in case of significant axillary burden before NAC should not be considered. Moreover, Ollila et al. (76) showed the great variability in the approach among different surgeons. 
Usually patients with pre NAC node positive disease undergo ALND with the associated risks in terms of comorbidities and with an unclear benefit in terms of survival. Due to the upcoming data on the high rate of pCR in specific molecular subtypes as triple negative breast cancer (TNBC) and Her2 positive, ALND is starting to be considered as an overtreatment, justifying the use of the SLNB $(71,73,77)$.

These considerations are supported by three prospective clinical trials: (I) the ACOSOG Z1071 trial (27); (II) SLNB in patients with BC before and after NAC (SENTINA) (78) and; (III) sentinel node biopsy after NAC in biopsy-proven node-positive BC: the SN FNAC Study (79).

The first study, involving 2,471 patients, investigated the feasibility of SLNB after NAC in clinically axillary node positive patients with a good response to treatment (73). The authors found an axillary pCR of $41 \%$ with a FNR from $10.8 \%$ to $12.6 \%$ related to the chemotherapy regimen administered. This value became lower if more than one sentinel node were excised and with the use of immunohistochemical (8.7\%). Only in a subgroup of patients a clip was placed in the positive node before treatment; when the node with the clip corresponded to the sentinel node the FNR reported by authors was $6.8 \%$.

Moreover, a meta-analysis including 3,398 patients, showed a similar pCR (39.2\%) with a FNR of $13 \%$, substantially overlapping the results of the previous study (73).

Another European multicentric study conducted in Sweden, enrolled 195 node positive patients who underwent NAC showing a FNR of $4 \%$ in case of more than one sentinel node excised (80).

The number of sentinel node removed was associated with treatment effect detected $(88 \%$ in case of 3 nodes excised). Also an UK study, the ROSCO trial, showed an advantage of removing more than one axillary nodes after NAC in patients clinically positive when treatment started.

To reduce the FNR, many authors tried to identify and excise the pre-NAC positive nodes. Rubio showed how marking the pre-treatment positive nodes and removing them is associated with a lower FNR of SLNB. Conversely, Barrio et al. described how identifying post treatment changes in the nodes at pathological report is a proof that previous positive nodes were removed. They found treatment changes in $94 \%$ of the previous positive nodes with post NAC pCR (81). The subgroup analysis related to molecular subtypes showed treatment effect in $96 \%$ of TNBC and HER 2 positive tumors compared to $83 \%$ of hormone receptor positive disease.

In summary, performing SLNB with double tracer (blue dye and 99 Technetium), removing more than one node, marking with a clip the positive node to check the presence during SLNB seem to make this technique accurate (73). In fact, Caudle et al. described how with all these recommendations the FNR of SLNB can reach 2\% (28).

Usually, the FNR considered acceptable in the adjuvant setting is $10 \%$ and the same value was borrowed as the cut off reference for SLNB after NAC (72).

To assess this item, Galimberti et al. (82) retrospectively analyzed the outcome of $396 \mathrm{BC}$ patients $\mathrm{cN} 0$ or $\mathrm{cN}+$ prior to NAC, cN0 after NAC and who received SLNB. ALND was performed only in case of positive sentinel node. After a median follow-up of 61 months, the five-year OS was $90.7 \%$ : $93.3 \%$ in patients $\mathrm{cN} 0$ before treatment, and $86.3 \%(\mathrm{P}=0.12)$ in initially $\mathrm{cN} 1 / 2$ patients. The authors showed a similar axillary disease rate in initially $\mathrm{cN} 0$ patients and in initially $\mathrm{cN} 1-2$ became $\mathrm{cN} 0$ after treatment with no statistically significant outcome differences. These data are concordant with those from IBCSG 23-01 (83) and ACOSOG Z001119 (3) trials in which no outcome differences were observed between the ALND and no-ALND.

In conclusion, the presenting nodal stage is not a limit for SLNB. In case of positive axillary nodes pre-NAC the addition of double tracer technique, the removal of more than two nodes and marking the pretreatment positive nodes may be advisable to reduce the FNR.

Actually NCCN current guidelines support the option to perform a SLNB in patients with positive axillary lymph nodes before treatment and $\mathrm{cN} 0$ after chemotherapy $(29,74,84)$.

If on one hand, we haven't yet data regarding regional recurrence after SLNB, on the other the presence of lymph nodal disease in the sentinel node (from ITC to macrometastasis) still requires ALND.

Two ongoing randomized trials will clarify the recommendations regarding ALND after NAC: (I) the NSABPB-51 (85) on patients with axillary positive nodes before treatment converted to clinically node negative; all the enrolled patients will be randomly assigned to lymph node radiation; (II) the Alliance A11202 trial (https:// clinicaltrials.gov/ct2/show/NCT01901094) on patients with biopsy proven axillary positive nodes before NAC and a positive SLNB after surgery; patients are assigned randomly to ALND or not and all of them will undergo nodal radiation. 
Table 1 Variables considered in more used nomograms

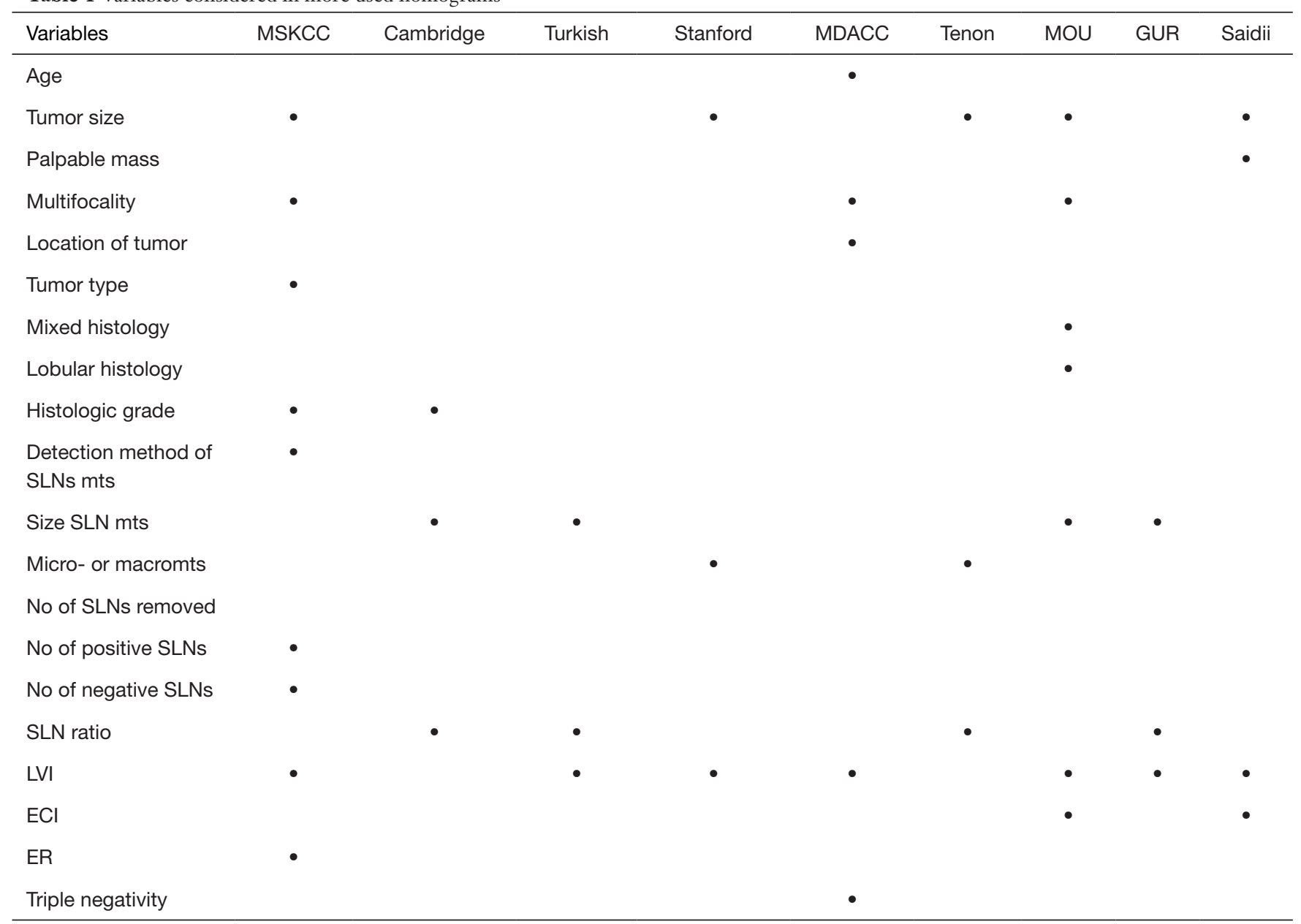

MSKCC, Memorial Sloan Kettering Cancer Center; MDACC, University of Texas MD Anderson Cancer Center; MOU, Masarykuv Onkologicky Ustav; SNLs, sentinel lymph nodes; mts, metastasis; SLN ratio, number of positive SLNs/total number of SLNs removed; LVI, lymphovascular invasion; $\mathrm{ECI}$, extracapsular invasion; ER, estrogen receptor.

With the increasing spread of neoadjuvant treatment, the greater rates of pCR and the trend towards more customized treatment planning, it is becoming crucial to have specific guidelines regarding loco-regional surgical treatment in this specific setting of patients.

\section{Nomogram and predictive factors of additive positive NSLNs}

Over the years, the parameters related to NSLNs metastasis risk after positive SLNB have been collected in nomogram, to recommend ALND and prevent local recurrence or to avoid unnecessary further surgery (Table 1).

Nowadays, some authors have tried to find out which nomogram may be more predictive.

Nadeem et al. (86) have compared the currently in use nomograms: Memorial Sloan Kettering Cancer Center (MSKCC), Masarykuv Onkologicky Ustav (MOU), university of Texas MD Anderson Cancer Center (MDACC), Cambridge, Turkish, Standford, Tenon. They constructed a ROC curve, calculate the AUC, finding in the MOU model the highest AUC value (0.74) and the lesser FNR for $\leq 10 \%$ probability $(0 \%$, but there were no patients in this low probability group).

Also Yildiz et al. (87) investigated the accuracy of nomogram and scoring system (MSKCC, MDACC, Tenon, Standford). They shared Nadeem's difficult to validate the MSKCC nomogram for the absence of size of SLNs 
metastasis, and found in no one of the five nomogram a good AUC discrimination. Instead, despite the limitation of a single and small population, a statically significant correlation was identified between multifocality and size of primary tumor and SLN metastasis in a multivariate logistic regression analysis.

In conclusion, Nadeem and Yildiz agree that nomograms lose their accuracy and good discriminatory power when performed in other centers, having different population from those from institution where they were developed. An accurate nomogram should include the most possible variables, including size of metastasis in SLNs that is not yet usually documented in the pathologic data sets.

Van den Hoven et al. (88) compared nine nomograms: MSKCC, Stanford, Mayo, Cambridge, GUR, MOU, SAIDI, Tenon, MDACC. They considered not only AUC but also calibration as an important parameter in predictive tool evaluation, finding best value for MSKCC nomogram, followed by Mayo model.

The authors specified that an individual approach in decision making is needed, since the allowed percentage of missing NLSNs metastasis differs from patient to patient, and predictive models of high probability of having more than three positive SLNB have to be developed.

Including its limitation, MSKCC nomogram is to date the more applied and supported in its validation by different retrospective analysis from single different institution $(89,90)$.

To date only Meretoja et al. (91) elaborated a nomogram based on multicentric international experience. They elaborated a formula that considers principal variables associated with additional positive NSLNs: percentage of patients with positive NSLNs, LVI, multifocality, HER2 status, number of negative and positive sentinel nodes, size of tumor, size of metastasis, EIC. This model has a good accuracy and calibration, both in internal and external applications, but it needs to be more validated before use it in clinical practice.

Freedman et al. (92), Gülben et al. (93) and Houvenaeghel et al. (94) have underlined there is a subgroup of patients that may need of ALND completion (about $15 \%$ of patients in Fredman's analysis). Parameters not included in the ACOSOG Z0011 criteria and in MSKCC nomogram could reveal these population: age, HR status, HER2 status, ki67 level, presence of LVI and/or ECI .

Also Fujii et al. (95) highlighted the importance of presence of ECI, finding a relation between ECI in SLNs and positive NLSN while disease recurrence is associated to
ECI only if is present in NLSN.

The European OSNA Users Committee (96) developed a new nomogram to predict, during operation, the risk of positive NLSN based on the CK19 mRNA copy determined by OSNA assay and tumor size (the other predictive factor, LVI, is unreliable in a preoperative core biopsy). According to this nomogram, an ALND is to perform for values higher than $31 \%$.

\section{Axillary management in elderly women}

The prime risk factor for $\mathrm{BC}$ is ageing, mostly affecting women age $>65$ years, $20 \%$ of which being over 75 (97). Despite the significant impact of this disease on the worldwide population, existing data supporting BC management during elderly are limited. The available information is often based on broad and randomized clinical trials that specifically exclude patients over 65 years of age (98). The lack of dedicated relevant data resulted in the actual unclear roadmap for an appropriate management of elderly BC (99). Elderly women are thought to have biologically more favorable tumors. The actual approach sees younger women more likely to be treated with BCS and SLNB, opposed to older patients most often treated with mastectomy and ALND (100). Some of the reasons why aggressive treatment of such patients are not often subscribed include extensive comorbidities, patients' preference, lack of social support, a declining functional and mental status, a minimal estimated life expectancy and physician preference (99). SLNB staging in elderly women is frequently omitted (101-104). Nodal staging by ALND, in the setting of positive SLNB, can influence both systemic adjuvant chemotherapy and loco-regional radiotherapy decisions. However, its impact on cancer specific survival and on the receipt of adjuvant therapy among older patients remains unclear (105) and cannot be so clear-cut, also considering the significant potential side effects associated with the ALND. Martelli et al. (106) found no differences in overall or breast-specific cancer survival at the 15 years follow-up stage between ALND and no-ALND groups, within older patients who had T1N0 breast-cancer, with a lower risk of axillary recurrence in the no-ALND group. Similarly, Javid et al. (105) found that 5 years all causes survival and $\mathrm{BC}$ specific survival did not differ between positive SLNB older patients who underwent SLNB only compared to the treatment SLNB + ALND. In the mentioned reference it has been identified a panel of factors such as higher age, advanced tumor size, undifferentiated 
historical grade, macro-regional nodal disease, negative hormone receptor status and mastectomy versus BCS, associated with a significantly higher risk of BC-specific mortality. The number of comorbidities was not associated with the receipt of ALND suggesting that age may play an independent role in surgical decision making. Aziz et al. (107) found a trend toward survival benefit among patients $>70$ years old who underwent ALND, becoming non-significant upon propensity analysis adjusting for receipt of ALND predictors. Several studies suggest that omitting completion of ALND not only in elderly patients with clinically N0 tumors (108), but also in positive SLNB patients (107) may be reasonable as it would have no impact on DFS and OS (104). Given the lack of direct benefits in the literature in terms of survival and local recurrence of the tumor, surgeons may choose to stage older women only when they have high suspicions of positive lymph nodes because many fragile elderly women may not benefit from lymph node staging, especially when their axilla is clinically normal (99). ALND in older patients may be reserved for cases in which data from ALND would clearly impact adjuvant therapy decisions.

\section{Conclusions}

Our review of the latest literature has confirmed the recent trend of de-escalation in the axillary surgical approach in $\mathrm{BC}$ with the goal of sparing unnecessary ALND even in the categories of patients traditionally subjected to ALND and reducing the associated morbidities. The indications and issues regarding the positive SLNB approach during conservative surgery and during mastectomy have become clearer, ongoing studies on these topics will provide more accurate information. In the positive SLNB setting, the adoption of the RT, according to the most important parameters, still remains a significant element in local control. About patients treated with NAC, the use of SLNB even in $\mathrm{cN}+$ pre-NAC patients appear to be useful and reliable in avoiding ALND in case of negative SLNB. Important studies are ongoing to evaluate the possibility of not performing ALND even in presence of positive SLNB.

Furthermore, the relevance of a more accurate evaluation of the preoperative axillary staging has been demonstrated while the SLNB intraoperative study is losing significance.

The axillary approach must therefore be re-evaluated in all categories of patients including the elderly who represent an ever increasing group of patients.

The aim of reducing surgery in axillary treatment is expressed by the presence of current studies that are considering the possibility of omitting every axillary approach in selected patient groups.

\section{Acknowledgments}

Funding: None.

\section{Footnote}

Provenance and Peer Review: This article was commissioned by the editorial office, Translational Cancer Research for the series "Update of Current Evidences in Breast Cancer Multidisciplinary Management". The article has undergone external peer review.

Conflicts of Interest: All authors have completed the ICMJE uniform disclosure form (available at http://dx.doi. org/10.21037/tcr.2017.09.19). The series "Update of Current Evidences in Breast Cancer Multidisciplinary Management" was commissioned by the editorial office without any funding or sponsorship. RM served as the unpaid Guest Editor of the series. The authors have no other conflicts of interest to declare.

Ethical Statement: The authors are accountable for all aspects of the work in ensuring that questions related to the accuracy or integrity of any part of the work are appropriately investigated and resolved.

Open Access Statement: This is an Open Access article distributed in accordance with the Creative Commons Attribution-NonCommercial-NoDerivs 4.0 International License (CC BY-NC-ND 4.0), which permits the noncommercial replication and distribution of the article with the strict proviso that no changes or edits are made and the original work is properly cited (including links to both the formal publication through the relevant DOI and the license). See: https://creativecommons.org/licenses/by-nc-nd/4.0/.

\section{References}

1. Veronesi U, Paganelli G, Viale G, et al. A randomized comparison of sentinel-node biopsy with routine axillary dissection in breast cancer. N Engl J Med 2003;349:546-53.

2. Reyna C, Lee MC, Frelick A, et al. Axillary burden of disease following false-negative preoperative axillary evaluation. Am J Surg 2014;208:577-81. 
3. Giuliano AE, McCall L, Beitsch P, et al. Locoregional recurrence after sentinel lymph node dissection with or without axillary dissection in patients with sentinel lymph node metastases: the American College of Surgeons Oncology Group Z0011 randomized trial. Ann Surg 2010;252:426-32.

4. Cools-Lartigue J, Meterissian S. Accuracy of axillary ultrasound in the diagnosis of nodal metastasis in invasive breast cancer: a review. World J Surg 2012;36:46-54.

5. Alvarez S, Anorbe E, Alcorta P, et al. Role of sonography in the diagnosis of axillary lymph node metastases in breast cancer: a systematic review. AJR Am J Roentgenol 2006;186:1342-8.

6. Sapino A, Cassoni P, Zanon E, et al. Ultrasonographicallyguided fine-needle aspiration of axillary lymph nodes: role in breast cancer management. Br J Cancer 2003;88:702-6.

7. Montgomery LL, Thorne AC, Van Zee KJ, et al. Isosulfan blue dye reactions during sentinel lymph node mapping for breast cancer. Anesth Analg 2002;95:385-8.

8. Krishnamurthy S, Sneige N, Bedi DG, et al. Role of ultrasound-guided fine-needle aspiration of indeterminate and suspicious axillary lymph nodes in the initial staging of breast carcinoma. Cancer 2002;95:982-8.

9. de Kanter AY, van Eijck CH, van Geel AN, et al. Multicentre study of ultrasonographically guided axillary node biopsy in patients with breast cancer. Br J Surg 1999;86:1459-62.

10. Deurloo EE, Tanis PJ, Gilhuijs KG, et al. Reduction in the number of sentinel lymph node procedures by preoperative ultrasonography of the axilla in breast cancer. Eur J Cancer 2003;39:1068-73.

11. Grube BJ, Giuliano AE. The current role of sentinel node biopsy in the treatment of breast cancer. Adv Surg 2004;38:121-66.

12. Boland MR, Prichard RS, Daskalova I, et al. Axillary nodal burden in primary breast cancer patients with positive pre-operative ultrasound guided fine needle aspiration cytology: management in the era of ACOSOG Z011. Eur J Surg Oncol 2015;41:559-65.

13. Hieken TJ, Trull BC, Boughey JC, et al. Preoperative axillary imaging with percutaneous lymph node biopsy is valuable in the contemporary management of patients with breast cancer. Surgery 2013;154:831-8; discussion 838-40.

14. Verheuvel NC, van den Hoven I, Ooms HW, et al. The Role of ultrasound-guided lymph node biopsy in axillary staging of invasive breast cancer in the post-ACOSOG Z0011 trial era. Ann Surg Oncol 2015;22:409-15.

15. Caudle AS, Kuerer HM, Le-Petross HT, et al. Predicting the extent of nodal disease in early-stage breast cancer. Ann Surg Oncol 2014;21:3440-7.

16. van Wely BJ, de Wilt JH, Francissen C, et al. Metaanalysis of ultrasound-guided biopsy of suspicious axillary lymph nodes in the selection of patients with extensive axillary tumour burden in breast cancer. Br J Surg 2015;102:159-68.

17. Pilewskie $M$, Jochelson $M$, Gooch JC, et al. Is preoperative axillary imaging beneficial in identifying clinically nodenegative patients requiring axillary lymph node dissection? J Am Coll Surg 2016;222:138-45.

18. Y L JT, McGowan K, Cooley G, et al. The role of ultrasound guided core biopsy of axillary nodes in predicting macrometastases and avoiding overtreatment outside ACOSOG Z0011 parameters. Breast 2015;24:57-61.

19. Gentilini O, Veronesi U. Abandoning sentinel lymph node biopsy in early breast cancer? A new trial in progress at the European Institute of Oncology of Milan (SOUND: sentinel node vs Observation after axillary UltraSouND). Breast 2012;21:678-81.

20. van Roozendaal LM, Vane MLG, van Dalen T, et al. Clinically node negative breast cancer patients undergoing breast conserving therapy, sentinel lymph node procedure versus follow-up: a Dutch randomized controlled multicentre trial (BOOG 2013-08). BMC Cancer 2017;17:459.

21. Assing MA, Patel BK, Karamsadkar N, et al. A comparison of the diagnostic accuracy of magnetic resonance imaging to axillary ultrasound in the detection of axillary nodal metastases in newly diagnosed breast cancer. Breast $\mathrm{J}$ 2017;23:647-55.

22. Arslan G, Altintoprak KM, Yirgin IK, et al. Diagnostic accuracy of metastatic axillary lymph nodes in breast MRI. Springerplus 2016;5:735.

23. Nakano Y, Noguchi M, Yokoi-Noguchi M. The roles of 18-F-FDG-PET/CT and US-guided FNAC in assessment of axillary nodal metastases in breast cancer patients. Breast Cancer 2017;24:121-7.

24. Garcìa Vicente AM, Soriano Castrejón A, Cruz Mora MÁ, et al. Dual time point 2-deoxy-2-[18F] fluoro-d-glucose PET/CT: Nodal staging in locally advanced breast cancer. Rev Esp Med Nucl Imagen Mol 2014;33:1-5.

25. Diego EJ, McAuliffe PF, Soran A, et al. Axillary staging after neoadjuvant chemotherapy for breast cancer: a pilot study combining sentinel lymph node biopsy with radioactive seed localization of pre-treatment positive axillary lymph nodes. Ann Surg Oncol 2016;23:1549-53. 
26. Koolen BB, Donker M, Straver ME, et al. Combined PET-CT and axillary lymph node marking with radioactive iodine seeds (MARI procedure) for tailored axillary treatment in node-positive breast cancer after neoadjuvant therapy. Br J Surg 2017;104:1188-96.

27. Boughey JC, Suman VJ, Mittendorf EA, et al. Sentinel lymph node surgery after neoadjuvant chemotherapy in patients with node-positive breast cancer: the ACOSOG Z1071 (Alliance) clinical trial. JAMA 2013;310:1455-61.

28. Caudle AS, Yang WT, Krishnamurthy S, et al. Improved axillary evaluation following neoadjuvant therapy for patients with node-positive breast cancer using selective evaluation of clipped nodes: implementation of targeted axillary dissection. J Clin Oncol 2016;34:1072-8.

29. National Comprehensive Cancer Network (NCCN) guidelines version 2.2017. Breast cancer.

30. Veronesi U, Paganelli G, Galimberti V, et al. Sentinel-node biopsy to avoid axillary dissection in breast cancer with clinically negative lymph-nodes. Lancet 1997;349:1864-7.

31. Banerjee SM, Michalopoulos NV, Williams NR, et al. Detailed evaluation of one step nucleic acid (OSNA) molecular assay for intra-operative diagnosis of sentinel lymph node metastasis and prediction of non-sentinel nodal involvement: experience from a London Teaching Hospital. Breast 2014;23:378-84.

32. Lyman GH, Somerfield MR, Giuliano AE. Sentinel lymph node biopsy for patients with early-stage breast cancer: 2016 American Society of Clinical Oncology Clinical practice guideline update summary. J Oncol Pract 2017;13:196-8.

33. Snow R, Reyna C, Johns C, et al. Outcomes with and without axillary node dissection for node-positive lumpectomy and mastectomy patients. Am J Surg 2015;210:685-93.

34. Milgrom S, Cody H, Tan L, et al. Characteristics and outcomes of sentinel node-positive breast cancer patients after total mastectomy without axillary-specific treatment. Ann Surg Oncol 2012;19:3762-70.

35. van der Noordaa MEM, Vrancken Peeters MTFD, Rutgers EJT. The intraoperative assessment of sentinel nodes - Standards and controversies. Breast 2017;34 Suppl 1:S64-9.

36. Ruano MA, Lopez-Bonet E, Buxó M, et al. An improved axillary staging system using the OSNA assay does not modify the therapeutic management of breast cancer patients. Sci Rep 2014;4:5743.

37. Babar M, Madani R, Jackson P, et al. One Step Nucleic Acid Amplification (OSNA) positive micrometastasis and additional histopathological NSLN metastasese: results from a single institution over 53 months. Surgeon 2016;14:76-81.

38. Nabais C, Figueiredo j, Lopes P, et al. Total tumor load assessed by one-step nucleic acid amplification assay as an intraoperative predictor for non-sentinel lymph node metastasis in breast cancer. Breast 2017;32:33-6.

39. Piñero-Madrona A, Ruiz-Merino G, Bernet L, et al. Tumoral load quantification of positive sentinel lymph nodes in breast cancer to predict more than two involved nodes. Breast 2014;23:859-64.

40. Peg V, Sansano I, Vieites B, et al. Role of total tumour load of sentinel lymph node on survival in early breast cancer patients. Breast 2017;33:8-13.

41. Robertson JF, Herrod PJ, Matthew J, et al. Treatment of the axilla in patients with primary breast cancer and low burden axillary disease: limitations of the evidence from randomised controlled trials. Crit Rev Oncol Hematol 2017;110:74-80.

42. Verheuvel NC, Voogd AC, Tjan-Heijnen VC, et al. Potential impact of application of Z0011 derived criteria to omit axillary lymph node dissection in node positive breast cancer patients. Eur J Surg Oncol 2016;42:1162-8.

43. Voutsadakis IA, Spadafora S. Recommendation for omitting axillary lymph node dissection should be individualized in patients with breast cancer with one or two positive sentinel lymph nodes. J Clin Oncol 2014;32:3901-2.

44. Dengel LT, Van Zee KJ, King TA, et al. Axillary dissection can be avoided in the majority of clinically node-negative patients undergoing breast-conserving therapy. Ann Surg Oncol 2014;21:22-7.

45. Ngui NK, Elder EE, Jayasinghe UW, et al. Relevance of the American College of Surgeons Oncology Group Z0011 trial to breast cancer in the Australian setting. ANZ J Surg 2013;83:924-8.

46. Bonneau C, Bendifallah S, Reyal F, et al. Association of the number of sentinel lymph nodes harvested with survival in breast cancer. Eur J Surg Oncol 2015;41:52-8.

47. Subhedar P, Stempel M, Eaton A, et al. Do the ACOSOG Z0011 criteria affect the number of sentinel lymph nodes removed? Ann Surg Oncol 2015;22 Suppl 3:S470-5.

48. Yao K, Liederbach E, Pesce C, et al. Impact of the American College of Surgeons Oncology Group Z0011 randomized trial on the number of axillary nodes removed for patients with early-stage breast cancer. J Am Coll Surg 2015;221:71-81.

49. Chung A, Gangi A, Mirocha J, et al. Applicability of the 
ACOSOG Z0011 criteria in women with high-risk nodepositive breast cancer undergoing breast conserving surgery. Ann Surg Oncol 2015;22:1128-32.

50. Wang J, Mittendorf EA, Sahin AA, et al. Outcomes of sentinel lymph node dissection alone vs. axillary lymph node dissection in early stage invasive lobular carcinoma: a retrospective study of the surveillance, epidemiology and end results (SEER) database. PLoS One 2014;9:e89778.

51. Giuliano AE, Ballman K, McCall L, et al. Locoregional recurrence after sentinel lymph node dissection with or without axillary dissection in patients with sentinel lymph node metastases: long-term follow-up from the American College of Surgeons Oncology Group (Alliance) ACOSOG Z0011 randomized trial. Ann Surg 2016;264:413-20.

52. Tsao MW, Cornacchi SD, Hodgson N, et al. A populationbased study of the effects of a regional guideline for completion axillary lymph node dissection on axillary surgery in patients with breast cancer. Ann Surg Oncol 2016;23:3354-64.

53. Jagsi R, Chadha M, Moni J, et al. Radiation field design in the ACOSOG Z0011 (Alliance) Trial. J Clin Oncol 2014;32:3600-6.

54. Donker M, van Tienhoven G, Straver ME, et al. Radiotherapy or surgery of the axilla after a positive sentinel node in breast cancer (EORTC 10981-22023 AMAROS): a randomised, multicentre, open-label, phase 3 non-inferiority trial. Lancet Oncol 2014;15:1303-10.

55. Sávolt Á, Péley G, Polgár C, et al. Eight-year follow up result of the OTOASOR trial: the optimal treatment of the axill—surgery or Radiotherapy after positive sentinel lymph node biopsy in early-stage breast cancer: a randomized, single centre, phase III, non-inferiority trial. Eur J Surg Oncol 2017;43:672-9.

56. Gooch J, King TA, Eaton A, et al. The extent of extracapsular extension may influence the need for axillary lymph node dissection in patients with T1-T2 breast cancer. Ann Surg Oncol 2014;21:2897-903.

57. Choi AH, Blount S, Perez MN, et al. Size of extranodal extension on sentinel lymph node dissection in the American College of Surgeons Oncology Group Z0011 trial era. JAMA Surg 2015;150:1141-8.

58. Reimer T, Stachs A, Nekljudova V, et al. Restricted axillary staging in clinically and sonographically nodenegative early invasive breast cancer (c/iT1-2) in the context of breast conserving therapy: first results following commencement of the Intergroup-SentinelMamma (INSEMA) trial. Geburtshilfe Frauenheilkd
2017;77:149-57.

59. Gentilini O, Botteri E, Dadda P, et al. Physical function of the upper limb after breast cancer surgery. Results from the SOUND (Sentinel node vs. Observation after axillary Ultra-souND) trial. Eur J Surg Oncol 2016;42:685-9.

60. Fu Y, Chung D, Cao MA, et al. Is axillary lymph node dissection necessary after sentinel lymph node biopsy in patients with mastectomy and pathological N1 breast cancer? Ann Surg Oncol 2014;21:4109-23.

61. Kenny TC, Dove J, Shabahang M, et al. Widespread Implications of ACOSOG Z0011: Effect on Total Mastectomy Patients. Am Surg 2016;82:53-8.

62. Miller CL, Specht MC, Skolny MN, et al. Risk of lymphedema after mastectomy: potential benefit of applying ACOSOG Z0011 protocol to mastectomy patients. Breast Cancer Res Treat 2014;144:71-7.

63. Cowher MS, Grobmyer SR, Lyons J, et al. Conservative axillary surgery in breast cancer patients undergoing mastectomy: long-term results. J Am Coll Surg 2014;218:819-24.

64. FitzSullivan E, Bassett RL, Kuerer HM, et al. Outcomes of sentinel lymph node- positive breast cancer patients treated with mastectomy without axillary therapy. Ann Surg Oncol 2017;24:652-9.

65. Tinterri C, Canavese G, Bruzzi P, et al. SINODAR ONE, an ongoing randomized clinical trial to assess the role of axillary surgery in breast cancer patients with one or two macrometastatic sentinel nodes. Breast 2016;30:197-200.

66. Goyal A, Dodwell D. POSNOC: a randomised trial looking at axillary treatment in women with one or two sentinel nodes with macrometastases. Clin Oncol (R Coll Radiol) 2015;27:692-5.

67. van Roozendaal LM, de Wilt JH, van Dalen T, et al. The value of completion axillary treatment in sentinel node positive breast cancer patients undergoing a mastectomy: a Dutch randomized controlled multicentre trial (BOOG 2013-07). BMC Cancer 2015;15:610.

68. de Boniface J, Frisell J, Andersson Y, et al. Survival and axillary recurrence following sentinel node-positive breast cancer without completion axillary lymph node dissection: the randomized controlled SENOMAC trial. BMC Cancer 2017;17:379.

69. Jatoi I, Benson JR, Toi M, et al. De-escalation of axillary surgery in early breast cancer. Lancet Oncol 2016;17:e430-41.

70. Mamounas E, Poulos C, Goertz HP, et al. Neoadjuvant Systemic Therapy for Breast Cancer: Factors Influencing Surgeons' Referrals. Ann Surg Oncol 2016;23:3510-7. 
71. Cain H, Macpherson IR, Beresford M, et al. Neoadjuvant therapy in early breast cancer: treatment considerations and common debates in practice. Clin Oncol (R Coll Radiol) 2017;29:642-52.

72. Rubio IT. Sentinel lymph node biopsy after neoadjuvant treatment in breast cancer: Work in progress. Eur J Surg Oncol 2016;42:326-32.

73. Ozmen T, Huff Vinyard A, Avisar E. Management of the positive axilla in 2017. Cureus 2017;9:e1216.

74. Pilewskie M, Morrow M. Axillary nodal management following neoadjuvant chemotherapy: a review. JAMA Oncol 2017;3:549-55.

75. Mamounas EP. Impact of neoadjuvant chemotherapy on locoregional surgical treatment of breast cancer. Ann Surg Oncol 2015;22:1425-33.

76. Ollila DW, Cirrincione CT, Berry DA, et al. Axillary management of stage II/III breast cancer in patients treated with neoadjuvant systemic therapy: results of CALGB 40601 (HER2-Positive) and CALGB 40603 (Triple-Negative). J Am Coll Surg 2017;224:688-94.

77. Dixon JM. Sentinel lymph node biopsy after neoadjuvant chemotherapy in patients with breast cancer. Breast Cancer Manag 2015;4:271-4.

78. Kuehn T, Bauerfeind I, Fehm T, et al. Sentinel-lymphnode biopsy in patients with breast cancer before and after neoadjuvant chemotherapy (SENTINA): a prospective, multicentre cohort study. Lancet Oncol 2013;14:609-18.

79. Boileau JF, Poirier B, Basik M, et al. Sentinel node biopsy after neoadjuvant chemotherapy in biopsy-proven nodepositive breast cancer: the SN FNAC study. J Clin Oncol 2015;33:258-64.

80. Zetterlund LH, Frisell J, Zouzos A, et al. Swedish prospective multicenter trial evaluating sentinel lymph node biopsy after neoadjuvant systemic therapy in clinically node-positive breast cancer. Breast Cancer Res Treat 2017;163:103-10.

81. Barrio AV, Mamtani A, Edelweiss M, et al. How often is treatment effect identified in axillary nodes with a pathologic complete response after neoadjuvant chemotherapy? Ann Surg Oncol 2016;23:3475-80.

82. Galimberti V, Ribeiro Fontana SK, Maisonneuve P, et al. Sentinel node biopsy after neoadjuvant treatment in breast cancer: five-year follow-up of patients with clinically nodenegative or node-positive disease before treatment. Eur J Surg Oncol 2016;42:361-8.

83. Galimberti V, Cole BF, Zurrida S, et al. International Breast Cancer Study Group Trial 23-01 investigators. Axillary dissection versus no axillary dissection in patients with sentinel-node micrometastases (IBCSG 23-01): a phase 3 randomised controlled trial. Lancet Oncol 2013;14:297-305.

84. Houssami N, Macaskill P, von Minckwitz G, et al. Metaanalysis of the association of breast cancer subtype and pathologic complete response to neoadjuvant chemotherapy. Eur J Cancer 2012;48:3342-54

85. Julian TB, Anderson SJ, Krag DN, et al. 10 year follow up of NSABP B-32, a randomized phase III clinical trial to compare sentinel node resection to conventional axillary dissection in clinically node negative breast cancer patients. J Clin Oncol 2013;31:100.

86. Nadeem RM, Gudur LD, Saidan ZA. An independent assessment of the 7 nomograms for predicting the probability of additional axillary nodal metastases after positive sentinel lymph node biopsy in a cohort of British patients with breast cancer. Clin Breast Cancer 2014;14:272-9.

87. Yildiz R, Urkan M, Hancerliogulları O, et al. Comparison of five different popular scoring systems to predict nonsentinel lymph node status in patients with metastatic sentinel lymph nodes: a tertiary care center experience. Springerplus 2015;4:651.

88. Van den Hoven I, Kuijt G, Roumen R, et al. A head to head comparison of nine tools predicting non-sentinel lymph node status in sentinel node positive breast cancer women. J Surg Oncol 2015;112:133-8.

89. Vieni S, Graceffa G, La Mendola R, et al. Application of a predictive model of axillary lymph node status in patients with sentinel node metastasis from breast cancer. A retrospective cohort study. Int J Surg 2016;35:58-63.

90. Bi X, Wang Y, Li M, et al. Validation of the Memorial Sloan Kettering Cancer Center nomogram for predicting non-sentinel lymph node metastasis in sentinel lymph node-positive breast-cancer patients. Onco Targets Ther 2015;8:487-93.

91. Meretoja TJ, Audisio RA, Heikkilä PS, et al. International multicenter tool to predict the risk of four or more tumorpositive axillary lymph nodes in breast cancer patients with sentinel node macrometastases. Breast Cancer Res Treat 2013;138:817-27.

92. Freedman GM, Fowble BL, Li T, et al. Risk of positive nonsentinel nodes in women with 1-2 positive sentinel nodes related to age and molecular subtype approximated by receptor status. Breast J 2014;20:358-63.

93. Gülben K, Berberoğlu U, Aydoğan O, et al. Subtype is a predictive factor of nonsentinel lymph node involvement in sentinel node-positive breast cancer patients. J Breast 
Cancer 2014;17:370-5.

94. Houvenaeghel G, Classe JM, Garbay JR, et al. Survival impact and predictive factors of axillary recurrence after sentinel biopsy. Eur J Cancer 2016;58:73-82.

95. Fujii T, Yajima R, Yamaguchi S, et al. Extracapsular invasion of sentinel lymph nodes is not associated with disease recurrence in breast cancer. Int Surg 2014;99:305-8.

96. Di Filippo F, Di Filippo S, Ferrari AM, et al. Elaboration of a nomogram to predict nonsentinel node status in breast cancer patients with positive sentinel node, intraoperatively assessed with one step nucleic amplification: retrospective and validation phase. J Exp Clin Cancer Res 2016;35:193.

97. National Cancer Institute (NCI). Surveillance, Epidemiology, and End Results (SEER) Program. Available online: https://seer.cancer.gov/

98. Hutchins LF, Unger JM, Crowley JJ, et al. Underrepresentation of patients 65 years of age or older in cancer-treatment trials. N Engl J Med 1999;341:2061-7.

99. Angarita FA, Chesney T, Elser C, et al. Treatment patterns of elderly breast cancer patients at two Canadian cancer centres. Eur J Surg Oncol 2015;41:625-34.

100. Grumpelt AM, Ignatov A, Tchaikovski SN, et al. Tumor characteristics and therapy of elderly patients with breastcancer. J Cancer Res Clin Oncol 2016;142:1109-16. 101. Weggelaar I, Aben KK, Warle MC, et al. Declined

Cite this article as: Terribile DA, Accetta C, D'Archi S, Paris I, Di Giorgio D, Garganese G, Fragomeni SM, Masetti R. Axillary lymph node surgical treatment. Transl Cancer Res 2018;7(Suppl 3):S390-S404. doi: 10.21037/tcr.2017.09.19 guideline adherence in older breast cancer patients: a population-based study in the Netherlands. Breast J 2011;17:239-45.

102. Rocco N, Rispoli C, Pagano G, et al. Undertreatment of breast cancer in the elderly. BMC Surg 2013;13:S26.

103. Joerger M, Thurlimann B, Savidan A, et al. Treatment of breast cancer in the elderly: a prospective, populationbased Swiss study. J Geriatr Oncol 2013;4:39-47.

104. Vetter M, Huang DJ, Bosshard G, et al. Breast cancer in women 80 years of age and older: a comprehensive analysis of an underreported entity. Acta Oncol 2013;52:57-65.

105. Javid SH, He H, Korde LA, et al. Predictors and outcomes of completion axillary node dissection among older breast cancer patients. Ann Surg Oncol 2014;21:2172-80.

106. Martelli G, Boracchi P, Ardoino I, et al. Axillary dissection versus no axillary dissection in older patients with T1N0 breast cancer: 15-year results of a randomized controlled trial. Ann Surg 2012;256:920-4.

107.Aziz D, Gardner S, Pritchard K, et al. Selective application of axillary node dissection in elderly women with early breast cancer. Ann Surg Oncol 2007;14:652-9.

108. Rudenstam CM, Zahrieh D, et al. Randomized trial comparing axillary clearance versus no axillary clearance in older patients with breast cancer: first results of International Breast Cancer Study Group Trial 10-93. J Clin Oncol 2006;24:337-44. 